\title{
ON SOME PROPERTIES OF DE PRIL TRANSFORMS OF \\ COUNTING DISTRIBUTIONS
}

\author{
BY BJøRN SUNDT
}

The Wyatt Company, Oslo

\begin{abstract}
In this paper we study a transform introduced by DE PRIL (1989) for recursive evaluation of convolutions of counting distributions with a positive probability in zero. We discuss some cases where the evaluation of this transform is simplified and relate the transform to infinitely divisible distributions. Finally we discuss an algorithm presented by DHAENE \& VANDEBROEK (1994) for recursive evaluation of convolutions.
\end{abstract}

\section{KEYWORDS}

Transforms ; recursions ; convolutions.

\section{INTRODUCTION}

1A. In Sundt (1992) we discussed the properties of a class of counting distributions $F$ whose discrete density $f$ satisfies a recursion in the form

$$
f(i)=\sum_{j=1}^{k}\left(a_{j}+\frac{b_{j}}{i}\right) f(i-j) \quad(i=1,2, \ldots)
$$

with $f(i)=0$ for $i<0$. We obviously must have $f(0)>0$. The distribution given by (1) was denoted by $R_{k}[\boldsymbol{a}, \boldsymbol{b}]$ with $\boldsymbol{a}=\left(a_{1}, \ldots, a_{k}\right)$ and $\boldsymbol{b}=\left(b_{1}, \ldots, b_{k}\right)$. Furthermore, we denoted by $\mathscr{R}_{k}$ the class of such distributions with a fixed number $k$ of terms in the recursion, and we introduced

$$
\mathscr{R}_{k}^{0}=\mathscr{R}_{k} \sim \mathscr{R}_{k-1} ; \quad(k=1,2, \ldots)
$$

the class $\mathscr{R}_{0}$ consists of the degenerate distribution concentrated in zero.

Expressing a distribution in the form $R_{k}[a, b]$ can in many cases simplify evaluation of convolutions and compound distributions, and such representations therefore seem to be of some interest.

In particular, any counting distribution $F$ with a positive probability in zero can be expressed in the form $R_{\infty}[\mathbf{0}, c]$, and thus $\mathscr{R}_{\infty}$ is the class of all such distributions. By solving

$$
f(i)=\frac{1}{i} \sum_{j=1}^{i} c_{j} f(i-j)
$$


with respect to $c_{i}$ we obtain

$$
c_{i}=\frac{1}{f(0)}\left[i f(i)-\sum_{j=1}^{i-1} c_{j} f(i-j)\right] ; \quad(i=1,2, \ldots)
$$

in this paper we interpret $\sum_{i=r}^{s}=0$ when $s<r$. From the way we constructed this recursion it is obvious that the sequence $c$ is uniquely determined. We shall call $c$ the De Pril transform of the distribution $F$. When we in the following discuss the De Pril transform of a distribution, it is silently assumed that the distribution belongs to $\mathscr{R}_{\infty}$.

The following theorem indicates the usefulness of De Pril transforms.

Theorem 1. The De Pril transform of the convolution of a finite number of distributions in $\mathscr{R}_{\infty}$ is the sun of the De Pril transforms of these distributions.

Theorem 1 was first proved by De Pril (1989) and restated in terms of the classes $\mathscr{R}_{k}$ by Sundt (1992).

From Theorem 1 we see that we can evaluate the convolution of a finite number of distributions in $\mathscr{R}_{\infty}$ by first evaluating the De Pril transform of each of the distributions by (3), then finding the De Pril transform of the convolution by summing the De Pril transforms of the individual distributions, and finally finding the discrete density of the convolution by (2).

As we have seen, a distribution is uniquely determined by its De Pril transform. However, unfortunately we cannot apply the recursion (2) directly to find a discrete density if we only know the De Pril transform as we also need the initial value $f(0)$. If $f$ has a finite support, then we can in principle start with an arbitrary value of $f(0)$ and then rescale afterwards so that the probabilities sum to one. However, the situation is much simpler when $f(0)$ is known, and this would normally be the case. For instance, when calculating convolutions by using (3), Theorem 1, and (2), we know that the probability in zero of the convolution is the product of the probabilities in zero of the original distributions.

It should be emphasised that when using Theorem 1 to evaluate convolutions, then the condition that each of the individual distributions should have a positive probability at zero, is not a serious restriction. If one of the distributions, $F$, has a positive probability in the integer $m \neq 0$ and probability zero in all integers less than $m$, then we can replace it with the shifted distribution $F_{0}$ given by

$$
F_{0}(i)=F(i-m) \quad(i=1,2, \ldots)
$$

and shift the resulting convolution the opposite way.

Unfortunately, calculating convolutions by Theorem 1 can sometimes be rather time-consuming as for each of the individual distributions we first have to evaluate the elements of its De Pril transform recursively by (3), and then perform the recursion (2) after having summed the $c_{i}$ 's from the different distributions. 
Therefore, De Pril (1989) suggested a less time-consuming approximation to the De Pril transform. Such approximations have been further studied by Dhaene \& De Pril (1994) and Dhaene \& Sundt (1994).

1B. In Section 2 of the present paper we shall deduce a recursive algorithm for evaluating the De Pril transform of distributions in the form $R_{k}[a, b]$ in terms of $a$ and $b$. If $k$ is small, this algorithm seems to be more efficient than using (3). When $k=1$, we can easily find simple explicit expressions for the elements of the De Pril transform from the algorithm of Section 2, and this is the topic of Section 3. Section 4 is devoted to De Pril transforms of compound distributions. Finally, in Section 5 we consider an alternative way of evaluating convolutions, recently presented by Dhaene \& Vandebroek (1994).

Some of the results that we prove in this paper, have been proved earlier, but we have included new proofs to relate the results to De Pril transforms.

\section{THE CLASSES $\mathscr{R}_{k}$}

2A. The following result can be applied for recursive evaluation of the De Pril transform of a distribution in $\mathscr{R}_{k}$.

Theorem 2. The De Pril transform $\boldsymbol{c}$ of the distribution $\mathscr{R}_{k}[\boldsymbol{a}, \boldsymbol{b}]$ satisfies the recursion

$$
c_{i}=i a_{i}+b_{i}+\sum_{j=1}^{k} a_{j} c_{i-j} \quad(i=1,2, \ldots)
$$

with $a_{i}=b_{i}=0$ for $i>k$ and $c_{i}=0$ for $i \leq 0$.

Proof. In Sundt (1992) it was shown that a distribution is in the form $R_{k}[a, b]$ if and only if the derivative of the natural logarithm of its probability generating function can be expressed as $\tilde{e}(s) s^{-1} /(1-\tilde{a}(s))$ with

$$
\tilde{a}(s)=\sum_{i=1}^{k} a_{i} s^{i} \quad \tilde{e}(s)=\sum_{i=1}^{k}\left(i a_{i}+b_{i}\right) s^{i}
$$

Thus

$$
\tilde{c}(s)=\sum_{i=1}^{\infty} c_{i} s^{i}
$$

has to satisfy

$$
\tilde{c}(s) s^{-1}=\frac{\tilde{e}(s) s^{-1}}{1-\tilde{a}(s)},
$$


which we rewrite as

$$
\tilde{c}(s)=\tilde{e}(s)+\tilde{a}(s) \tilde{c}(s) .
$$

By expressing the right-hand side as a power series and comparing coefficients with the left-hand side, we obtain (4).

Q.E.D.

As some of the quantities in (4) are equal to zero, this formula can be rewritten as

$$
\begin{gathered}
c_{i}=i a_{i}+b_{i}+\sum_{j=1}^{i-1} a_{j} c_{i-j} \quad(i=1,2, \ldots, k) \\
c_{i}=\sum_{j=1}^{k} a_{j} c_{i-j} . \quad(i=k+1, k+2, \ldots)
\end{gathered}
$$

We see that (7) is a homogeneous linear difference equation of order $k$ with constant coefficients. In principle it can be solved by using the values of $c_{1}, \ldots, c_{k}$ as constraints when $k<\infty$. However, for numerical evaluation it would normally be more efficient to use the recursive form (6)-(7).

2B. From Theorems 1 and 2 we easily obtain the following result, which was also proved in Sundt (1992).

Theorem 3. The convolution of the $n$ distributions $R_{k}\left[a, b^{(r)}\right](r=1, \ldots, n)$ is $R_{k}[a, b]$ with

$$
b_{i}=(n-1) i a_{i}+\sum_{r=1}^{n} b_{i}^{(r)} . \quad(i=1, \ldots, k)
$$

Proof. Let $\boldsymbol{c}^{(r)}$ denote the De Pril transform of $R_{k}\left[a, b^{(r)}\right](r=1, \ldots, n)$ and $c$ the De Pril transform of their convolution. Then

$$
\begin{aligned}
& c_{i}=\sum_{r=1}^{n} c_{i}^{(r)}=\sum_{r=1}^{n}\left[i a_{i}+b_{i}^{(r)}+\sum_{j=1}^{k} a_{j} c_{i-j}^{(r)}\right]= \\
& i a_{i}+(n-1) i a_{i}+\sum_{r=1}^{n} b_{i}^{(r)}+\sum_{i=1}^{k} a_{i} c_{i-j}=i a_{i}+b_{i}+\sum_{j=1}^{k} a_{j} c_{i-j},
\end{aligned}
$$

which proves the theorem.

Q.E.D.

By letting $k=\infty$ and $\boldsymbol{a}=0$ in Theorem 3 , we obtain Theorem 1 .

2C. In Sundt (1992) we proved the following theorem.

Theorem 4. A distribution on the range $\{0,1, \ldots, k\}$ with a positive probability at zero and discrete density $f$ can be expressed as $R_{k}[a, b]$ with

$$
a_{i}=-\frac{f(i)}{f(0)} \quad b_{i}=2 i \frac{f(i)}{f(0)} . \quad(i=1, \ldots, k)
$$


By inserting (9) in (4) we obtain (3). Hence, by applying Theorem 4 to express a distribution in the form $R_{k}[a, b]$ we simply obtain the original recursion (3) for the De Pril transform and no simplification.

\section{THE CASE $k=1$}

When the distribution is in the form $R_{1}[a, b],(6)$ and (7) reduce to

$$
\begin{aligned}
& c_{1}=a+b \\
& c_{i}=a c_{i-1}, \quad(i=2,3, \ldots) .
\end{aligned}
$$

from which we obtain

$$
c_{i}=(a+b) a^{i-1} . \quad(i=1,2, \ldots)
$$

The following theorem is proved in Sundt \& Jewell (1981).

Theorem 5. The distribution $R_{1}[a, b]$ is binomial if $a<0$, Poisson if $a=0$, and negative binomial if $a>0$.

Let us look at (10) in the three cases described in Theorem 5.

\section{i) Binomial}

$$
f(i)=\left(\begin{array}{l}
t \\
i
\end{array}\right) \pi^{i}(1-\pi)^{t-i} . \quad(i=0,1, \ldots, t ; \mathfrak{t}=1,2, \ldots ; 0<\pi<1)
$$

Then

$$
\begin{gathered}
a=-\frac{\pi}{1-\pi} \quad b=\frac{\pi}{1-\pi}(t+1) \\
c_{i}=-t\left(\frac{\pi}{\pi-1}\right)^{i} . \quad(i=1,2, \ldots)
\end{gathered}
$$

Formula (11) illustrates a disappointing aspect of application of Theorem 1 for calculating convolutions. Although $f(i)=0$ for all $i>t, c_{i} \neq 0$ for all values of $i$, even for $i>t$, and if $\pi>\frac{1}{2}$, then $c$ is unbounded.

\section{ii) Poisson}

$$
f(i)=\frac{\lambda^{i}}{i !} e^{-\lambda} . \quad(i=0,1, \ldots ; \lambda>0)
$$


Then

$$
\begin{array}{rl}
a=0 & b=\lambda \\
c_{i}=\lambda \delta_{1, i}, \quad & (i=1,2, \ldots)
\end{array}
$$

where $\delta_{i, j}$ denotes the Kronecker delta. This simple result is not surprising. Already from Theorem 5 we had that the Poisson distribution could be represented in the form $R_{1}[0, b]$. Only the degenerate distribution concentrated in zero has a simpler De Pril transform than the Poisson distribution; for the degenerate distribution all elements of the De Pril transform are equal to zero.

\section{iii) Negative binomial}

$$
f(i)=\left(\begin{array}{c}
\alpha+i-1 \\
i
\end{array}\right)(1-\pi)^{\alpha} \pi^{i} . \quad(i=0,1, \ldots ; \alpha>0 ; 0<\pi<1)
$$

In that case

$$
\begin{gathered}
a=\pi \quad b=(\alpha-1) \pi \\
c_{i}=\alpha \pi^{i} . \quad(i=1,2, \ldots)
\end{gathered}
$$

We see that unlike the binomial distribution, the negative binomial distribution will always have a bounded De Pril transform.

\section{COMPOUND DISTRIBUTIONS}

4A. The following theorem is proved in Sundt (1992).

Theorem 6. A compound distribution in $\mathscr{R}_{\infty}$ with counting distribution $R_{k}[a, b]$ and severity distribution with discrete density $h$ can be expressed as $R_{\infty}[d, c]$ with

$$
d_{i}=\frac{\sum_{j=1}^{k} a_{j} h^{j^{*}}(i)}{1-\sum_{j=1}^{k} a_{j} h(0)^{j}} \quad c_{i}=\frac{i \sum_{j=1}^{k} \frac{b_{j}}{j} h^{j^{*}}(i)}{1-\sum_{j=1}^{k} a_{j} h(0)^{j}}, \quad(i=1,2, \ldots)
$$

To find the De Pril transform of the compound distribution, one can first evaluate $\boldsymbol{d}$ and $\boldsymbol{c}$ by (14) and then use Theorem 2 to find the De Pril transform. However, a natural question is whether one could arrive more directly from the De Pril transform of the counting distribution if this transform is known. The following corollary to Theorem 6 expresses the De Pril transform of the compound distribution in terms of the De Pril transform of the counting distribution and the discrete density of the severity distribution and is obtained from Theorem 6 by putting $k=\infty$ and $\boldsymbol{a}=\mathbf{0}$. 
Corollary 1. A compound distribution in $\mathscr{R}_{\infty}$ with counting distribution $R_{\infty}[\mathbf{0}, b]$ and severity distribution with discrete density $h$ has De Pril transform $c$ with

$$
c_{i}=i \sum_{j=1}^{\infty} \frac{b_{j}}{j} h^{j *}(i) . \quad(i=1,2, \ldots)
$$

We have silently assumed that the sum in (15) (as well as the sums in the numerators in (14) when $k=\infty$ ) converges to a finite value. In the following we shall discuss some cases where the summand in this sum differs from zero only for a finite number of values of $j$, and then this condition is obviously fulfilled. In particular we see that if $h(0)=0$, then $h^{j^{*}}(i)=0$ for $i<j$, and (15) reduces to

$$
c_{i}=i \sum_{j=1}^{i} \frac{b_{j}}{j} h^{j^{*}}(i) . \quad(i=1,2, \ldots)
$$

4B. We now consider the case

$$
h(i)=\delta_{m, i} \quad(i=0,1, \ldots)
$$

for some positive integer $m$, that is, all severities are equal to $m$ with probability one. Then

$$
h^{j^{*}}(i)=\delta_{(j m), i .} \quad(i=0,1, \ldots ; j=1,2, \ldots)
$$

Insertion in (15) gives that

$$
c_{k m}=m b_{k} \quad(k=1,2, \ldots)
$$

and $c_{i}=0$ for all other values of $i$.

The relation (18) can be applied if we want to find the De Pril transform of an arithmetic distribution with span $m>1$. In this case we first rescale the distribution so that it obtains span one, then we find the $b_{i}$ 's, e.g. by (3) or Theorem 2 , and finally we find the $c_{i}$ 's by (18).

It is interesting to compare (18) with evaluation of the De Pril transform $d$ of the $m$-fold convolution of $R_{\infty}[\mathbf{0}, \boldsymbol{b}]$. From Theorem 1 follows that

$$
d_{i}=m b_{i}, \quad(i=1,2, \ldots)
$$

that is, we get the same elements as in the case when $m$ is a scaling factor, but in the latter case these elements are more "spread out".

4C. Let us now consider the case when the counting distribution is the Poisson distribution presented in Section 3 , that is, $R_{1}[0, \lambda]$. In that case (15) reduces to

$$
c_{i}=i \lambda h(i) . \quad(i=1,2, \ldots)
$$

We shall deduce a characterisation of compound Poisson distributions in terms of De Pril transforms. For that purpose we shall need the following lemma. 
Lemma 1. A distribution in $\mathscr{R}_{\infty}$ with a non-negative De Pril transform $c$ satisfies the condition

$$
\sum_{i=1}^{\infty} \frac{c_{i}}{i}<\infty
$$

Proof. Let $f$ be the discrete density of a distribution in $\mathscr{R}_{\infty}$ with a non-negative De Pril transform $c$. From (3) we obtain for $i=1,2, \ldots$

$$
\frac{c_{i}}{i}=\frac{1}{f(0)}\left(f(i)-\frac{1}{i} \sum_{j=1}^{i-1} c_{j} f(i-j)\right) \leq \frac{f(i)}{f(0)}
$$

as the sum is obviously non-negative. Summation over $i$ gives

$$
\sum_{i=1}^{\infty} \frac{c_{i}}{i} \leq \sum_{i=1}^{\infty} \frac{f(i)}{f(0)}=\frac{1-f(0)}{f(0)}<\infty
$$

Theorem 7. A distribution in $\mathscr{R}_{\infty}$ is a compound Poisson distribution if and only if its De Pril transform is non-negative.

Proof. From (19) we immediately see that the De Pril transform of a compound Poisson distribution is non-negative.

Now let us consider a distribution in $\mathscr{R}_{\infty}$ with a non-negative De Pril transform $c$. From Lemma 1 we see that

$$
\lambda=\sum_{i=1}^{\infty} \frac{c_{i}}{i}<\infty
$$

Then the function $h$ defined by

$$
h(i)=\frac{c_{i}}{i \lambda} \quad(i=1,2, \ldots)
$$

is non-negative and satisfies the condition $\sum_{i=1}^{\infty} h(i)=1$. Thus $h$ is the discrete density of a probability distribution on the non-negative integers, and as $c$ satisfies (19), $c$ is the De Pril transform of a compound Poisson distribution with Poisson parameter $\lambda$ and severity distribution on the non-negative integers with discrete density $h$.

This completes the proof of Theorem 7 .

Q.E.D.

From Theorem 1 and (19) we easily obtain the following well-known result.

Theorem 8. The convolution of $n$ compound Poisson distributions with Poisson parameter $\lambda^{(r)}$ and discrete severity density $h^{(r)}$ on the non-negative integers $(r=1$, 
$\ldots, n)$ is a compound Poisson distribution with Poisson parameter

$$
\lambda=\sum_{r=1}^{n} \lambda^{(r)}
$$

and discrete severity density

$$
h=\frac{1}{\lambda} \sum_{r=1}^{n} \lambda^{(r)} h^{(r)}
$$

Proof. Let $c^{(r)}$ denote the De Pril transform of the $r$ th compound distribution $(r=1, \ldots, n)$ and $c$ the De Pril transform of the convolution. Then

$$
c_{i}=\sum_{r=1}^{n} c_{i}^{(r)}=\sum_{r=1}^{n} i \lambda^{(r)} h^{(r)}(i)=i \lambda h(i)
$$

that is, $\boldsymbol{c}$ is the De Pril transform of a compound Poisson distribution with Poisson parameter $\lambda$ and discrete severity density $h$. This proves the theorem. Q.E.D.

Now let $f$ denote the discrete density of the compound distribution. If $h(0)=0$, then $f(0)=e^{-\lambda}$, that is,

$$
\lambda=-\ln f(0) .
$$

Furthermore $h$ is given by (20). In general, also if $c$ does not represent a compound Poisson distribution, we can always define a quantity $\lambda$ by (21) and a function $h$ by (20), so that the distribution formally looks like a compound Poisson distribution. This has motivated Hürlimann (1990) to call distributions in $\mathscr{R}_{\infty}$ pseudo compound Poisson distributions. The function $h$ is a proper discrete probability density if and only if $f$ is the discrete density of a compound Poisson distribution.

4D. A distribution $F$ is called infinitely divisible if for all integers $n$ there exists a distribution $F_{n}$ such that $F$ is the $n$-fold convolution of $F_{n}$. It can be shown (cf. e.g. Feller (1968, Section XI.2)) that a distribution in $\mathscr{R}_{\infty}$ is infinitely divisible if and only if it can be expressed as a compound Poisson distribution. Combining this result with Theorem 7 gives the following characterisation of infinitely divisible distributions in $\mathscr{R}_{\infty}$ in terms of De Pril transforms, which was proved by Katti (1967).

Theorem 9. A distribution in $\mathscr{R}_{\infty}$ is infinitely divisible if and only if its De Pril transform $c$ is non-negative.

By applying Theorem 9 together with (13) and (11), we see that negative binomial distributions are infinitely divisible whereas binomial distributions are not. 
4E. Let us now consider the case when the counting distribution is a Bernoulli distribution with probability. In this case

$$
\begin{gathered}
\pi=1-f(0) \\
h(i)=\frac{f(i)}{1-f(0)}, \quad(i=1,2, \ldots)
\end{gathered}
$$

that is, $\pi$ is the probability that the compound variable is positive, and $h$ is the discrete density of the conditional distribution of this variable given that it is positive.

Insertion of (11) in (16) gives

$$
c_{i}=-i \sum_{j=1}^{i} \frac{1}{j}\left(\frac{\pi}{\pi-1}\right)^{j} h^{j^{*}}(i) . \quad(i=1,2, \ldots)
$$

Thus we have now obtained an explicit expression for $c_{i}$ that holds for all distributions in $\mathscr{R}_{\infty}$. Formula (22) was deduced by De Pril (1989).

4F. With a life assurance policy we often have the situation that when a claim occurs, then it has a fixed amount $m$. We assume that $m$ is a positive integer. Let $\pi$ be the probability that a claim occurs.

We are now in the frame-work of the previous subsection with $h$ given by (17) and obtain

$$
c_{k m}=-m\left(\frac{\pi}{\pi-1}\right)^{k} \quad(k=1,2, \ldots)
$$

and $c_{i}=0$ for all other values of $i$.

This model has been studied by De Pril (1986).

\section{The Dhaene-VAndebroek ALGORIthm}

5A. We want to evaluate the discrete density $f$ of the convolution of $n$ distributions in $\mathscr{R}_{\infty}$ with discrete density $f^{(r)}$ and De Pril transform $c^{(r)}(r=1, \ldots, n)$. Let $c$ denote the De Pril transform of the convolution. By Theorem 1

$$
c=\sum_{r=1}^{n} c^{(r)}
$$

As described in Section 1 , we can evaluate $f$ recursively by evaluating $c_{i}^{(r)}$ recursively by (3) for each $i$, summing

$$
c_{i}=\sum_{r=1}^{n} c_{i}^{(r)}
$$

and finally evaluating $f(i)$ by (2). 
Let us look a bit more closely at the two last steps. By insertion of (23) in (2) and interchanging the order of summations we obtain

$$
f(i)=\frac{1}{i} \sum_{r=1}^{n} d_{i}^{(r)} \quad(i=1,2, \ldots)
$$

with

$$
d_{i}^{(r)}=\sum_{j=1}^{i} c_{j}^{(r)} f(i-j) . \quad(i=1,2, \ldots ; r=1, \ldots, n)
$$

For convenience we also introduce $d_{i}^{(r)}=0$ for $i \leq 0$.

Dhaene \& Vandebroek (1994) have deduced the following algorithm for recursive evaluation of the $d_{i}^{(r) \text { 's }}$ and shown that in many situations $f$ can be evaluated more efficiently by using this algorithm together with (24) than by applying the procedure described above.

Theorem 10. The $d_{i}^{(r)}$ 's defined by (25) can be evaluated recursively by

$$
d_{i}^{(r)}=\frac{1}{f^{(r)}(0)} \sum_{j=1}^{i}\left[j f(i-j)-d_{i-j}^{(r)}\right] f^{(r)}(j) . \quad(i=1,2, \ldots)
$$

5B. In Theorem 2 we showed that $c^{(r)}$ could be evaluated more efficiently than by (3) if the distribution belonged to $\mathscr{R}_{k}$ when $k$ is small. The following theorem gives an analogous result for the $d_{i}^{(r)}$, s.

Theorem 11. If $f^{(r)}$ is the discrete density of $R_{k}[a, b]$, then

$$
d_{i}^{(r)}=\sum_{j=1}^{k}\left[\left(j a_{j}+b_{j}\right) f(i-j)+a_{j} d_{i-j}^{(r)}\right] . \quad(i=1,2, \ldots)
$$

Proof. We introduce the power series

$$
\begin{gathered}
\tilde{a}(s)=\sum_{i=1}^{k} a_{i} s^{i} \quad \tilde{e}(s)=\sum_{i=1}^{k}\left(i a_{i}+b_{i}\right) s^{i} \\
\tilde{c}^{(r)}(s)=\sum_{i=1}^{\infty} c_{i}^{(r)} s^{i} \\
\tilde{d}^{(r)}(s)=\sum_{i=1}^{\infty} d_{i}^{(r)} s^{i} \\
\tilde{f}(s)=\sum_{i=1}^{\infty} f(i) s^{i} .
\end{gathered}
$$

From (5) we see that

$$
\tilde{c}^{(r)}(s)=\tilde{e}(s)+\tilde{a}(s) \tilde{c}^{(r)}(s)
$$


and from (25) we obtain

$$
\tilde{d}^{(r)}(s)=\tilde{c}^{(r)}(s) \tilde{f}(s)
$$

By multiplying (28) by $f(s)$ and inserting (29) we obtain

$$
\tilde{d}^{(r)}(s)=\tilde{e}(s) \tilde{f}(s)+\tilde{a}(s) \tilde{d}^{(r)}(s),
$$

and by comparing coefficients in the power series expansions of both sides of (30) we obtain (27).

Q.E.D.

Analogous to Theorem 2, when determining $\boldsymbol{a}$ and $\boldsymbol{b}$ by Theorem 4, (27) brings us back to (26).

5C. Let us now consider the special case when $f^{(r)}$ is the discrete density of $R_{k}\left[\boldsymbol{a}, \boldsymbol{b}^{(r)}\right](r=1, \ldots, n)$. Application of (24) and (27) gives

$$
\begin{gathered}
f(i)=\frac{1}{i} \sum_{r=1}^{n} d_{i}{ }^{(r)}=\frac{1}{i} \sum_{r=1}^{n} \sum_{j=1}^{k}\left[\left(j a_{j}+b_{j}^{(r)}\right) f(i-j)+a_{j} d_{i-j}^{(r)}\right]= \\
\frac{1}{i} \sum_{j=1}^{k}\left[\left(n j a_{j}+\sum_{r=1}^{n} b_{j}^{(r)}\right) f(i-j)+a_{j} \sum_{r=1}^{n} d_{i-j}^{(r)}\right]= \\
\frac{1}{i} \sum_{j=1}^{k}\left[\left(n j a_{j}+\sum_{r=1}^{n} b_{j}^{(r)}\right) f(i-j)+a_{j}(i-j) f(i-j)\right]=\sum_{j=1}^{k}\left(a_{j}+\frac{b_{j}}{i}\right) f(i-j),
\end{gathered}
$$

with $\boldsymbol{b}$ given by (8), that is, we obtain the defining recursion (1) of $R_{k}[\boldsymbol{a}, \boldsymbol{b}]$. Thus we have found yet another proof of Theorem 3 .

In particular, if the $n$ distributions are identical and $\boldsymbol{a}$ and $\boldsymbol{b}^{(1)}$ are determined by Theorem 4, then Theorem 10 and (24) give De Pril's (1985) recursion for the $n$-fold convolution of a distribution in $\mathscr{R}_{\infty}$. This has also been pointed out by Dhaene \& Vandebroek (1994).

5D. Unlike $c^{(r)}$, the $d_{i}^{(r)}$ 's do not only depend on $f^{(r)}$, but also on the other distributions. If we were only to evaluate the convolution of $n$ given distributions, this is not a drawback. However, if we also want to evaluate the convolution of other combinations of the same distributions, then we can immediately apply the De Pril transforms that we have already evaluated, for evaluating the convolutions by Theorem 1 and formula (3).

\section{ACKNOWLEDGEMENT}

The present research was carried out while the author stayed at the Department of Mathematics at the Katholieke Universiteit Leuven with support from the Research Council of that university. The author is grateful to Jan Dhaene for interesting discussions which led to improvements of the paper. 


\section{REFERENCES}

DE PrIL, N. (1985) Recursions for convolutions of arithmetic distributions. ASTIN Bulletin 15, 135-139.

DE PrIL, N. (1986) On the exact computation of the aggregate claims distribution in the individual life model. ASTIN Bulletin 16, 109-112.

DE PRIL, N. (1989) The aggregate claims distribution in the individual model with arbitrary positive claims. ASTIN Bulletin 19, 9-24.

Dhaene, J. and De PrIL, N. (1994) On a class of approximative computation methods in the individual risk model. Insurance: Mathematics and Economics 14, 181-196.

DhAENE, J. and SUNDT, B. (1994) On approximating distributions by approximating their De Pril transforms. Onderzoeksrapport 9427. Departement Toegepaste Economische Wetenschappen, Katholieke Universiteit Leuven.

DhaEnE J. and VANDEBroeK, M. (1994) Recursions for the individual model. To be published in Insurance: Mathematics and Economics.

FELLER, W. (1968) An introduction to probability theory and its applications. Vol. 1 (3rd ed.). Wiley, New York.

HürlımanN, W. (1990) Pseudo compound Poisson distributions in risk theory. ASTIN Bulletin 20, 57-79.

KATTI, S. K. (1967) Infinite divisibility of integer-valued random variables. Annals of Mathematical Statistics 38, 1306-1308.

SundT, B. (1992) On some extensions of Panjer's class of counting distributions. ASTIN Bulletin 22, $61-80$.

SUNDT, B. and JEWELL, W.S. (1981) Further results on recursive evaluation of compound distributions. ASTIN Bulletin 13, 27-39.

\section{BJøRN SUNDT}

The Wyatt Company A.S

P.O. Box 1508 Vika

N-0117 Oslo, Norway 